SLAC-PUB -4335

DEB? 011597

$S L A C-P U B-4335$

May 1987

(A)

$$
\text { CONF- } 870623.8-1
$$

\title{
THE NUMERICAL SIMULATION OF ACCELERATOR COMPONENTS ${ }^{*}$
}

\author{
W. B. Herrmannsfeldt and H. Manerfeld \\ Stanford Linear Accelerator Center \\ Stanford University, Stanford, California, 9/905
}

\begin{abstract}
The techniques of the numerical simulation of plasmas can be readily applied to problems in accelerator physics. Because the problems usually involve a single component "plasma," and times that are at most, a few plasma oscillation periods, it is frequently possible to make very good simulations with relatively modest computation resources.

We will discuss the methods and illustrate them with several examples. One of the more powerful techniques of understanding the motion of of charged particles is to view computer-generated motion pictures. We will show several little movie strips to illustrate the discussions. The examples will be drawn from the application areas of Heavy Ion Fusion, electron-positron linear colliders and injectors for free-electron lasers.
\end{abstract}

Lecture given at the 1987 IEEE Minicourse on Computer Application in Plasma Scinnce, Washington, D.C., June 3-5, 1987

* Work supported by the Department of Energy, contract DE - AC03-765F005 15.

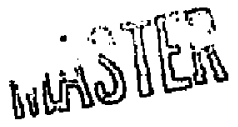




\section{Initroduction}

As we come to the last paper in this minicourse, you might reasonably expect that a paper on the simulation of accelerator components would demonstrate how the latest state-of-the-art simulation techniques can be applied to real problems. No such luk! I don't know if will disappoint the students, or make them feel superior, but the truth is that I am as interested as anyone is in learning these new techniques. The programs and methods that are generally used are mostly quite old. They range from the gun design program EGUN, that I started writing in 1962, and PARMILA which began as an even older MURA program in the '50's, to the magnet and rf cavity programs, POISSON and SUPERFISH, from the '60's and early '70's, to the various adaptations of the 2-D particle-in-cell (PIC) programs of the last decade. I think it is significant that the field of accelerator physics did not do very much of its own code development for a few years. Hopefully that is now changing; the new products, like the 3-D programs that are in this afternoon's schedule, are only just starting tc be used.

Partly the reasons for this lag between inventions and their utilization are the same as the reasons for the long time needed to deploy any new technology; nuclear power and superconductivity are two that come to mind. Partly it probably has to do with the way our laboratories are organized. It is the new projects, whether at new centers like CEBAF, or at established centers like LBL, that bring out the needs for new computational tools. Three such areas are: high-current injectors for free elertron lasers (FEL), heavy ion accelerators for Inertially Confined Fusion (HIF), and new TeV-range electron-positron colliders.

The above new projects have in common a central theme; all require intense beams of charged particles, approaching the space charge limit. Also, all three require extremely good beam quality. A partial list of components that can be simulated by the methods described during this course, and that are required for projects in the above three areas, is shown in I'able I.

\section{DISCLAIMER}

\footnotetext{
This report was prepayed as an actount of work sponsored by an agency of the Uniled States Government Neither the Unites States Governisent nor any agensy thereol, nor any of then employees, makes any warzanty, express or implied, or assumes any legal liability of responsi. bility for the accuracy. completeness, or usefulness of any information, apparatus, product, or process disclosed, or represents that its use would not infringe privalely owned rights. Refer. ence herein to any specific commencial product. process, or serviee by trade name, irademark. manulacturer, or otherwise docs not nesessarily constitule of imply its endorsemem, feoommendation. or favoring by the United Stales Government or any agency thereof. The views and opinions of authors expressed herein do not necessarily state or reflect those of the United Siales Govemment of any agency theroor.
} 


\section{TABLE I}

Some examples of accelerator components that can be modelled using the computational methods of plasma physics.

1. INJECTION SYSTEMS:
(a) The SLAC Linear Collider (SLC).
(b) The Boeing Free Electron Laser.
(c) The TRW-Stanford Superconducting FEL.
(d) The LANL laser photocathudr gun and injector.
(e) The four bean heavy ion injector for HIF at LANL and LBL.
(f) The "Relativistic Klystron" at SLAC and LLNL.
(g) The gun for the FEL at the Univ. of California at Santa Barbara.
(h) Electron guns for electron cooling at CERN and FERMUAB.

2. KLYSTRON AND LASERTRON RF POWER AMPLIFIERS:
(a) The $65 \mathrm{MW}$ S-band kjystron for the SLC.
(b) The S-band lasertron proof-of-principal test at SLAC.
(c) The high frecuency ribbon beam lasertron at Texas Accelerator Center.
(d) The $30 \mathrm{MW} \mathrm{X}$-band klystron at SLAC.
(e) Ribbon beans klystrons for high frequency and high power at several places.
(f) The gyroklystron at the Univ. of Maryland.

3. BEAM TRANSPORT:
(a) The transport of intense beams of heavy ions for HIF at LBL.
(b) The column design for the Santa Burbara FEL.
(c) The $2 \mathrm{MV}$ four-beam heavy ion injector for HIF at LBL.

4. FOCUSING SYSTEMS:
(a) The anal transport of heavy ion beams to the target pellet at LLNL and LBL.
(b) Beam disruption at the final focus for electron-positron linear colliders at SLAC.


The list in Table I could grow incefinitely, but it should be long cnough to give some favor to the type and range of applications of interest here. The locations named are centers that are or have worked on each project. Other laboratories have the same or similar projects in almost every case. For the rest of this talk we will try to expand briefly on one case from each of the four areas. It will be seen that the methods used are quite different for each example.

\section{Space-charge Limited Beam Transport}

This first example is from the work of Irving Haber ${ }^{1}$ at NRL. The objective is to find the space charge limit for transport in a quadrupole focused beam line. There are several reasons to be interested in this example;

1. It shows that the PIC code method is capable of doing much more than just sirnulating an accelerator component; it can actually do physics. Of course, experimental confirmation is still required. In this case, the key experiments were performed as part of the HIF program by Tiefenbach" ${ }^{2}$.

2. It addresses a basic problem in accelerator physics; how much current can be transported without instabilities that damage the emittance.

3. By yielding encouraging results, this work greatly enhanced the potential economic attractiveness of HIF.

The itundamental physics question is illustrated by $F$ ig. 1 . In the absence of space charge, (top figure) a single particle exercises betatron motion through $2 \pi$ radians in a certain length, $L$, measured as the number (probably not an integer) of lattice periods of the focusing quadrupole lenses. (The quadrupoles may be either magnetic or electrostatic elements.) The phase advance per period is then $\sigma_{o}=2 \pi / L$.

In the presence of space charge, the added defocusing force results in a longer betatron wave length, and consequently a new phase advance $\sigma$, which is lower. It is clear that sigma cannot be less than zero for stable transport, because that implies an expanding beam. Therefore, the measure of stability is to determine how close to zero the phase advance can be depressed without encountering growth in the emittance of the beam.

There are three ways to attempt to answer thie space charge limited transport question;

1. Through theory with envelope equations. This was done by Lambertson, et al $^{3}$, using the Kapchinskij-Vladimirskj (K-V) distribution, which because it is uniform in all projections through phase space, results in linear forces which can be treated analytically. 
2. Numerically using particle-in-cell techniques.

3. Experimentally by building a lonz quadrupole transport system.

There are difficulties with each method. The analytic approach can find the threshold for instabilities, but cannot show whether they are unique to the distribution, nor to what extent they can damage the beam. The numerical simulation also depends on initial distributions, but can use several different ones. Because at the lowest tune shift, fairly small effects are being examined, it is necessary to use large numbers of particles and fine cell structures. (Neither of these are fundamental difficulties.) The experiments require great care to show that the limitation is not due to the initial emittance of the source, or to nonlinearities in the focusing system, or other experimental errors such as alignment, etc.

The historical sequence was as follows; the analytic calculations were made starting with the beginning of the Heavy Jon Fusion program in 1976. They showed various regions of instability, in which different modeg would grow, up to the regior shown cross hatched in Fig. 2. In this region, there are always different modes growing, sometimes simultaneously, so that stable transport seems impossible. The numerical simulations were made next, motivated at least in part by the need to investigate the isolated regions of instability that were suspected to depend upon the initial distribution. While a complete review of these studies is beyond the scope of this paper, it is worthwhile to note the general conclusion that a space charge limited beam somewhat redistributes itself very quickly. Depending on the initial emittance, this redistribution may represent an increase in the emittance which may then remain stable, or grow only very slowly. As the strength of the focusing elements is increased, which is the natural adjustment to increase the current carrying capacity of the system, there comes a point where the single particle tune approaches $\sigma_{0}=90^{\circ}$. This region can be seen in the confirming experimental data shown in the cluster of points on Tig. 2. The experimental data all lie above the line determined by the quality of the beam from the ion source, and below the line for $\sigma=\sigma_{p}$, which corresponds to zero current.

Figure 3 gives the results of a numerical simulation, from Reference 2, for a case in which the space charge has depressed the phase advance from $\sigma_{\theta}=90^{\circ}$ to $\sigma=15^{\circ}$. The summary of the emittance growth shown in Fig. 3 comes from a series of calculations of the type shown in Fig. 4, which is a single frame from a film strip that will be shown to the class. We will view several cases with different currents, all with $\sigma_{\rho}=90^{\circ}$. Typical simulations in this series used 16384 particles in a $128 \times 128$ cell system. Tests with higher numbers of particles were made to assure that numerical effects were not responsible for the enittance growth that 
was observed. Time and space reyuitc thit we leave this stibject at this point.

\section{High Power Klystrons}

The next three areas that I will cover all relate to work at SLAC. The first two, high power klystrons and rf buncher systems for injectors, were the applications that initially caused us to turn to PIC code techniques. The third area, which is the interaction area of a high energy electron-positron linear collider, sequires such specialized physics that, although PIC code techniques are used, the programs are very specific to the application,

We have applied the 2-D PIC code MASK ${ }^{4}$ to the problems of modelling high power klystrons. The methods used were devised by Siman $\mathrm{Yu}^{5}$. Very briefly, the criteria for a successful simulation require including several key features;

1. It must be capable of handling the full range of signals from the small signals in the input region, ( $\mathrm{a}$ few tens of watts in the presence of a multimegawatt electron beam) to the large signals in the output end of a klystron.

2. It must deal with the complex particle trajectories in the output region, up to and including particles which are stopped and turned back.

3. It must correctly treat the beam dynamics including all the self-field effects.

4. It must be aole to correctly handle particles from low energies, through the trans-relativistic range, up to very relativistic energies. Similarly, all of the field equations must be relativistic.

5. It must be three dimensional in particle motion because the transverse velocities, both radial and azimuthal, are so important in the beam dynamics of a slystron. It is usually sufficient to consider only azimuthally symmetric fields.

The ultimate objective of this work is to design the components for the TeVrange linear collider of the future. However, in order to establish credibility, our initial goal was to significantly improve our modelling capability for the present generation of klystrons. SLAC has been in the process of replacing all 240 klystrons on the linac as part of the energy upgrade needed to obtain greater than $50 \mathrm{GeV}$ from a linac that was originally designed for $20 \mathrm{GeV}$.

Rather than to go into details about the design and performance of these tubes, about which you can read elsewhere ${ }^{6}$, I want to turn to one special problem that was solved with the aid of the computer simulation. The tubes being built exhibited a tendency toward severe amplitude modulation of the output pulse, in the range of 10-20 $\mathrm{MHz}$. 'This phenomenon depended critically on the setting 
of the focusing coils, in particular, on the strength of the coil that establishes an opposing field in the region of the cathode. In principal, this coil allows one to establish the degree to which the tube is to be operated in "immersed" flow, as opposed to having zero field on the cathode, which is the usual accelerator gun requirement.

Now it is very undesirable to have such a critical setting apply to each tube. Even more important, it was necessary to identify the cause of the amplitude modulation. There was a great deal of speculation about this, in particular, why the beam dynamics of the gun should have such an effect on the output. Eventually a tube was built with a special coating of moly-ruthenium on the edge of the cathode. This material is normally applied to the back of the cathode to inhibit any emission from the back side. Adding the coating to the side was done to attempt to inhibit edge emission. Although not totally solving the problem, this tube was more stable than others without this treatment. This led to modelling the beam dynamics associated with edge emission.

In contrast to many of the other simulations that are made of accelerator components using electromagnetic PIC codes, the klystron is primarily of interest at steady state. Thus one must find a way to achieve a satisfactory degree of steady state operation with a reasonable amount of computer time. The device that has been used is to simulate the high- $Q$ cavities with external equivalent circuit expressions that define the phase and amplitude of an if voltage that is applied across a special boundary or "port" in the wall of a simulated tube. The If Gelds are first allowed to build up, and then particles are injected and allowed to pass through the device for a few rf cycles. Since, in the real klystron the flelds are induced in the cavities by the beam, and in turn induce still more growth in the $\mathrm{r}$ current in the beam, the key element in this simulation is the link beiween the rf currents and the beam dynamics. The phase and amplitude of the induced If currents are related to the real and imaginary parts of the volume integral of $\vec{E} \cdot \vec{J}$, where $\vec{E}$ and $\vec{J}$ are the electric field and current density, respectively in the vicinity of an if cavity. Aided by certain simplificatione that result from considering the way $\mathrm{z}$. klystron operates in steady state, $\mathrm{Yu}^{\mathrm{E}}$ has developed a set of efficient algorithms that are able to model a complete six-cavity tube in ten to twelve CRAY computer runs.

An example of the output from the simulation of the SLAC 5045 lilystron is shown in Fig. 5. The beam is introduced from the left using the output from the electrostatic electron gun program $\mathbf{E G U N}^{7}$. The if cavities are located where the slots are shown in the side wall. The situation shown in Fig. 5 is a single snapshot of a moment during an if cycle. We will show a pair of film. strips that demonstrate how the if currents grow as the particles pass through 
the klystron. It is worthwhile first. to point out that the longitudinal bunebing, which is what actually makes a klystron function, is characterized more by radial bulges and dips than it is by longitudinal compression of the charge. It is not a bad approximation at these current levels to consider the sipace charge as an incompressible fluid.

Returning now to the subject of the amplitude mooulation, and its link to the edge emission from the cathode, we next examine a simulation of the electron gun shown in Fig. 6. In this figure, electrons have been allowed to start by space charge limited flow from a cathode that has been extended to cover a roughly one millimeter wide slot between cathode and focus electrode. The model for this behavior is the observation that there is a very large area of hot emitter just behind the slot. The slot can thus be a virtual cathode capable of the same space charge limited emission dessity as any part of the solid cathode. In fact, the area of the slot is approximately $5 \%$ of the area of the catnode, and including this area accounts for upwards of half of the roughly $10 \%$ discrepancy between the calculated and observed pervennce of these tubes.

In the motion pictr res that we will view, Ken Eppley ${ }^{8}$, who has succeeded Simon Yu in this work, has coiored the electrons from the edge of the cathode yellow, while the rest of the bearn is colored tlue. As we have repeatedly stressed the importance of the transverse beam dynamics of the klystron, it is not surprising to find that the yellow electrons occupy a different bit of transverse phase space than does the rest of the beam. At one point during the rf cycle, the yellow electrons are found to go directly into the next-to-last if cavity. An expanded view of this part of the tube is shown in Fig. 7 with tracks from the simulation passing directly into the cavity.

As the second film strip will show, it is possible to find a setting for the magnetic field near the gun such that the beam does not enter the If cavity. This corresponds to the condition usually found experimentally in which for a narrow range of field settings, it is possible to obtain stable operation. It is not difficult to imagine a mechanism by which spraying a beam of electrons into a high field cavit $y^{\prime}$, would cause the fields within that cavity to collapse, leading to a relatation oscillation. This conjecture is supported by the observation that the characteristic period of the amplitude modulation corresponds to roughiy the natural time constant lor building up fields in these klystrons, in the range of a few ten: of namoseconds, for a frequency of approximately $20 \mathrm{MHz}$.

Neither the simulations, nor the fabrication tolerances, are accurate enough r,o expect precisely juantitative results from a study of this type. Especially this is true because the simulation is for steady state operation, while the effect keing examined violates steady state. Nevertheless, one trusts small changes 
in the simulation once the general characteristics of the behavior agree with observation. In this instance, numerical simulation has served as a valuable tool for some detective work in identifying the source of an annoying instability.

\section{RF Bunching System}

There is a great deal of similarity between the physics of if bunching for a linac injector, and the physics of amplifying the rf currents in a klystron amplifier. Prior to the introduction of the use of PIC codes for simulating rf bunchers, the most sophisticated programs available were the several variations of PARMELA ${ }^{9}$. These programs are still in general use, but are limited in their ability to properly simulate the very high intensity bunchers needed for newer applications such as for FEL's and linear colliders. It is interesting to note that the single bucket charge, and the emittance needed, are both approximately the same for the high intensity visible light FEL's and for the SLAC Linear Collider (SLC)i

The original purpose of the SLAC work with MASK was to test the feasibility of using the particle-in-cell method to simulate the injector for the SLC. The buncher component was modelled first, giving results consistent with actual operation $^{10}$. The model was enlarged so that eventually it included the second $178.5 \mathrm{MHz}$ sisharmonic buncher, the $2856 \mathrm{MHz}$ buncher and four accelerating cells. This model was used to study the emittarce and bunching characteristics of a pulse under conditions comparable to usage. The diagnostic results of these simulations indicated that the pulse was being over-focused in the subharmonic buncher. In an attempt to improve bunching, we departed Irom actual operating parameters by altering the externa! magnetic field. This proved to be helpful, resulting in more charge per pulse, higher peak amplitude, shorier pulse time, with comparable emittance growth.

MASK has proven to be a powerful toof enabling us to better understand the phenomena which take place within the injector. This is made possible because, in addition to quantitative results from MASK, the many diagnostics available allow the user to obtain visual images of the particie pulse as it progresses through the injector. These are represented by particle density and phase space plots of the pulse. Field and current plots fill in the visual picture.

The SLC injector is shown in Fig. 8. The plot is half of a cylindrical cross section with the center line of the cylinder along the bottom of the plot. The dar\} shaded areas along the top edge are ports at which electric fields are generated. This device is used instead of attempting to actually propagate fields along the length of the accelerator structure. The of fields are generated at the ports by specifying an if voltage across each port. The phase and amplitude of the voltage 
at each port determine the fields in the model. The fields are ramped up slowly over several rf cycles to avoid introducing sf urious components. The parameters for the simulation, including the rf voltages on the ports, are shown in Table II.

Table II.

MASK Simulation Parameters for the SLC Buncher

Pulse at Entry to $178.5 \mathrm{MHz}$ Subharmonic Buncher

Beam Radius

Beam Voltage

Current Width

Charge
$0.94 \mathrm{~cm}$

$114-196 \mathrm{keV}$

$1.75 \mathrm{~ns}$

$11.5 \mathrm{nC}$

Mask Setup

\begin{tabular}{|c|}
\hline $\begin{array}{l}\mathrm{d} \times 1 \\
\mathrm{~d} \times 2 \\
\text { rf cycle } \\
\text { Port Voltages }\end{array}$ \\
\hline 1 \\
\hline 2 \\
\hline 3 \\
\hline 4 \\
\hline 5 \\
\hline 6 \\
\hline 7 \\
\hline 8 \\
\hline 9 \\
\hline
\end{tabular}

$1.36773 \mathrm{ps}$

$0.972 \mathrm{~mm}$

$1.048 \mathrm{~mm}$

$256 \mathrm{dt}$

$68.4 \mathrm{kV}$

$41.5 \mathrm{kV}$

$41.5 \mathrm{kV}$

$41.5 \mathrm{kV}$

$41.5 \mathrm{kV}$

$376.2 \mathrm{kV}$

$376.2 \mathrm{kV}$

$376.2 \mathrm{kV}$

$376.2 \mathrm{kV}$ 
The actual buncher uses a solenoidal magnetic field for focussing the bearn. The rnagnetic field used in this simulation was generaied by defining a set of ideal coils to give an equivalent magnetic field.

Due to the length of the 'njector, it is not possible to simulate the eniire system with MASK at one time. Recent additions to the code would allow modelling the imjector in sections and using the output from one section as input to the next. In this simulation, the shape, charge and energy distributions of the pulse used as ingut to the second subharmonic buncher were derived from regults previously obtained by Mary James ${ }^{11}$.

The If buncher is based on creating a traveling wave that matches the velocity of th $\mathrm{beam}$. To generate the proper traveling wave within the buncher, the phase difference of two successive ports is $2 \pi / 3$. We are free to choose the phases of the first ports of the buncher and accelerator, as well as the phase of the port of the subharmonic buncher.

Bunching is produced by slowing down the particies in the front of the pulse and speeding up the particles in its rear. This is accomplished by arranging the time at which the pulse arrives at the ports to roincide with the proper phase of the rf field. For the port of the subharmonic buncher, we can obtain this result either, by changing the time at which injection begine or ky changing the initial phase of the port.

Figure 9 is a composite of eighteen particle density plots of a pulse at different

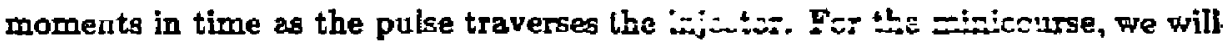
view a motion pictare strip showing the bunching process. The pulse is injected on the left and the bunchea pulse leaves at the right. The stairgtep contoin of the front and rear of the pulse on injection is a step-wise approximation to the desired elliptic shape. The current distribution was obtained by giving all the macro particles the same charge and wejght, while populating each stairstep with the particles in a K-V distribution. 


\section{Disruption in the Final Focus of a Linear Collider}

With the completion of the electron-positron storage ring LEP at CERN, colliding beams for electron-positron physics in the future must turn to the concept known as linear colliders. Very simple economics shows that the cost of energy for a linear accelerator must be a linear function of energy (above some small nominal value) ind due to the cost of $\mathrm{rf}$ power to replace energy lost by synchrotron radiation, the cost of sturage rings scales by at least the square of the energy. Thus no matter what the coefficient is, eventually the exponent wins and the linear accelerator must be the less costly option.

It is well known that there is a current limit for colliding beams in a storage ring, given by the small tune shift that can be tolerated as the bearns collide. This limits the luminosity in storage rings. This effect is absent for colliding linacs since there is usually no need for any concern about the quality of the bearr after the collision. The exceptions to this rule are that the disrupted beam must not strike the focusing elements as it leaves the interaction point, and thet in some examples of designs for linear colliders, it is hoped to direct the beam into the opposing linac in order to decellerate it and cxtract the rf power. We will not consider either of these probjems bere, although the problems of the exiting beam are quite real. Our concern is rather the disruption itself and how it may onhance or reduce the useful lumirasity of a future linear collider.

Studies of the disruption effects for colliding beams were made by Hollebeek ${ }^{12}$. He used an adaptation of particle-in-cell methods, called cloudi-in-cell, to obtain improved accuracy for the very high forces encountered in a linear collider. Some feeling for the problem can be obtained by considering some of the numbers:

1. Each pulse for the SLC should contain $5 \times 10^{10}$ electrons and an equal number of positrons.

2. The final spot size should be about 2 microns in radius, and the bunch should be about 100 microns long.

3. For the collider of the future, in order that the luminosity should increase proportional to the energy, the radius of the spot should be reduced to about 0.01 microns.

4. Considering the relativistic mass of each particle, the density in the final collision for the SLC approaches one tenth the density of water. For the TeV-range collider of the future, it approximates the density of iron.

Figure 11 shows uni somputer simulated collision generated by Hollebeek. The first effect of the - wilision is to cause the bunches to pinch due the the fact that the self magnotic fields come together in such a way as to add, which 
the space charge of the oppositely charged particles cancels. The effect for the parameter range for the SLC is to enhance the luminosity.

For colliders of the future, Chen and Yokoya ${ }^{13}$ have continued the disruption studies. Interesting effects arise when the magnetic field become so great that classical theory of synchrotron radiation would predict a critical energy for the photons emitted to be greater than the particle energy. The magnetic fields can be of the order of $10^{3}$ Tesla, for bunches of about $5 \times 10^{9}$ particles at $0.5 \mathrm{TeV}$ for each bearn. Under these conditions it is necessary to go to a quantum theory of the synchrotron radiation. in this case, a Monte Carlo technique is used to define when (on which time step) and at what eriergy, a photon is emitted. The concern is that the radiated energy so reduces the energy of the colliding particles, that the available center of mass energy is too low for the physicg intended. Also, the large energy spread complicates the problems of data anelyses.

One solution for this difficulty is to use a flat or ribbon beam. The aspect ratio of the ribbon is set to control the disruption while maintaining the desired luminosity. Here the numbers are equally impressive;

1. Energy; $0.5+0.5 \mathrm{TeV}$

2. Number of particles; $4.5 \times 10^{9}$ each positrons and electrons.

3. $\sigma_{x}=0.038 \mu \mathrm{m}$

4. $\sigma_{\mathrm{y}}=0.002 \mu \mathrm{m}$

5. $\sigma_{\mathrm{d}}=20 \mathrm{\mu m}$

\section{Conclusivn}

The computation methods of plasme physics have many applications in the field of accelerator physics. We have chosen a few examples with the intent of showing the range and power of these techniques.

\section{Acknowledgements}

The authors wish to express their gratitude so Irving Habei (NRL), and Ken Eppley and Pisin Chen (SLAC) for providing material used for the examples. They also want to express their appreciation to Adam Drobot, Larry Seftor and other staff members of the Science Appiication International Corapany (SAIC) for their advice and support in the use of MASK. 


\section{References}

1. Irving Haber, "Numerical Study of Space Charge Limited Transport in a Quadrupole Transport System with 90 Degree Phase Advance," NRL Memorandum Report 4474, Naval Research Laboratory, Washington, D.C., May 1981.

2. Michacl G. Tiefenback, "Space-Charge Limit on the Transport of Ion Beams in a Long Alternating Gradient System," LBL-22465, Lawrence Berkeley Laboratory, Berkeley, CA, November 1986.

3. G. Jambertson, L. J. Laslett, L. Smith, "Transport of Intense Ion Beams," IEEE Trans. Nucl. Sci., Yol. NS26, p. 993, June 1977.

4. A. Palevsiky and A. T. Drobot, "Application of E-M P.I.C. Codes to Microwave Devices," Proceedings of the Nirth Conference on Numerical Simulation of Plasmas, Fyanston, Itinois, 1980.

5. Simon Yu, "Paticle in Cell Simulation of High Power Klystrons," SLAC AP-34, Staniord Linear Accelerator Center, Stanford, California, September 1984.

6. G. T. Konrad, "High Power RF Klystrons for Linear Colliders," 1984 Linear Accelerator Conference, Seeheim/Darmstadt, West Germany, (SLACPUB-3324), May 1984.

7. W. B. Herrmannffeldt, "Election Trajectory Program," SLAC-22\%, Stanford Linear Accelerator Center, Stanford, CA 94305, November 1979.

8. K. R. Eppley, "MASK Simulation of Cathode Side E1oission," Unpublished internal memorandums, March 1986.

9. PARMELA is foind in various forms in many laboratories, and is mostly undocumented. Its roots go back to the program PARMILA, which was a MURA program written for proton linacs. Most of the recent development of PARMELA (the " $\mathrm{e}$ " in the name is for electrons) was made in the AT Division of Los Alamos National Laboratory.

10. H. Hanerfeld, W. B. Herrmannsfeldt, M. B. James, and R. H. Miller, SLC Injector Modeling, 1985 Particle Accelerator Conference, Vnncouver, British Columbia, May 13-16, 1985.

11. M. B. James and R. H. Miller, "A High Current Injector for the Proposed SLAC Linear Collider," IEEE Trans. Nucl. Sci, NS-28, p 3461, June 1981.

12. R. Hollebeek, "Disruption Limita for Linear Colliders," Nuclear Instruments and Methods 184, p 333-347, 1981.

13. P. Chen and $K$. Yokoya, private communication. 


\section{Figure Captions}

1. The betatron wavelength for a single particle in a quadrupole transport system depends on the amount of space charge in the beam.

2. The extent to which the phase advance per focusing period, $\sigma$, can be depressed towards zero without introducing emittance growth, is a measure of the current carrying capacity of a quadrupole system. The experimental results are from Tiefenbach's Ph.D. Thesis ${ }^{2}$.

3. The growth of emittance as a function of path length, measured in the number of focusing cells, or magnet doublets, for one of the cases calculated by Haber'.

4. A single frame from a computer generated motion picture for the case summarized by the plot in Fig. 3. Phase space projections are shown for the transverse coordinates $x, y, p_{x}$ and $p_{y}$. The number of filaments, six in this case, correspond to the mode or combination of modes, that is causing the emittance to grow.

5. A particle density plot from a single spapshot irom the simulation of the SLAC $65 \mathrm{MW}$ S-band klystron. The gaps along the top of the frame represent if ports which are driven according to equivalent circuit aigorithms. The output cavity is located just to the right of the last block; the change in radius is made to reproduce a corresponding change in the radius of the real klystrons just at the entrance to the collector.

6. An electron gun simulation for the SLAC $65 \mathrm{MW}$ klystron showing the trajectories that can be taken by electrons emitted from the gap between the cathode and the focus electrode.

7. An expanded view of the particle density plot of Fig. 5 showing that at a particular of phase angle, electrons are flowing directly into the nextto-last if cavity, which is the bunching cavity for a high power ilystron. The color coded computer generated motion pictures show that ihe edgeemitted electrons are the ones that end up in this cavity under most focusing conditions.

8. The cross section of the buncher for the SLC. The dark shaded lines along the upper edge of the figure represent of ports across which an of voltage is imposed to create the fields in the buncher.

9. Particle density plots from MASK for the SI burcher. The beam enters from the left with bunch length and energy profile as determined by a separate simulation of the prebuncher. 
10. Computer simulated collision of intense relativistic beams, illustrating the pinch effect, (from Hollebeek ${ }^{12}$ ). 
SPACE-CHARGE DEPRESSES THE BETATRON PHASE ADVANCE PER CELL

\section{WTTHOUT SPACE CHARGE}

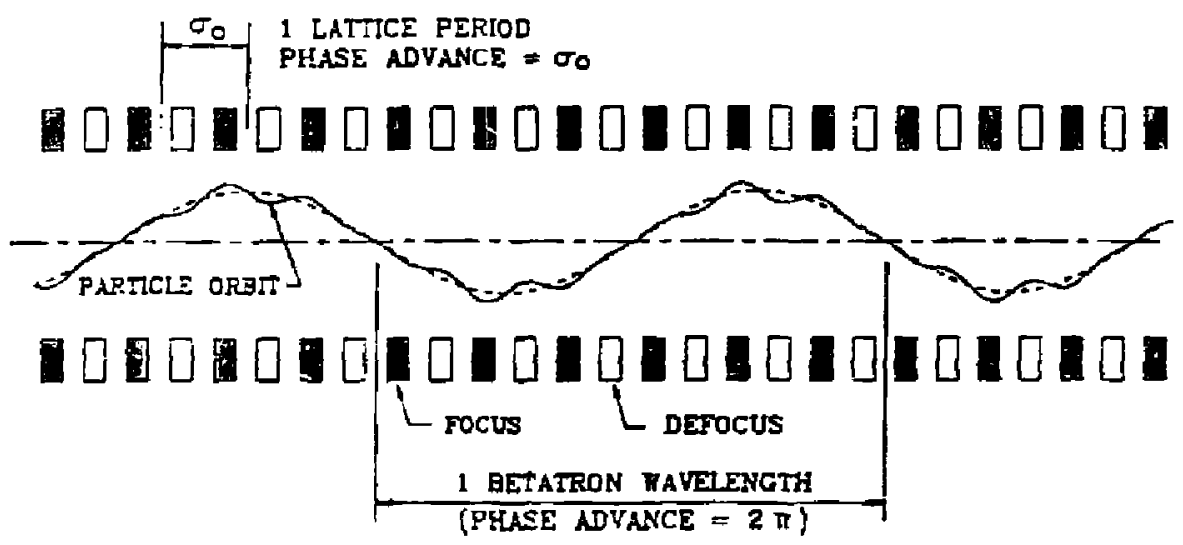

WTH SPACE CHARGE

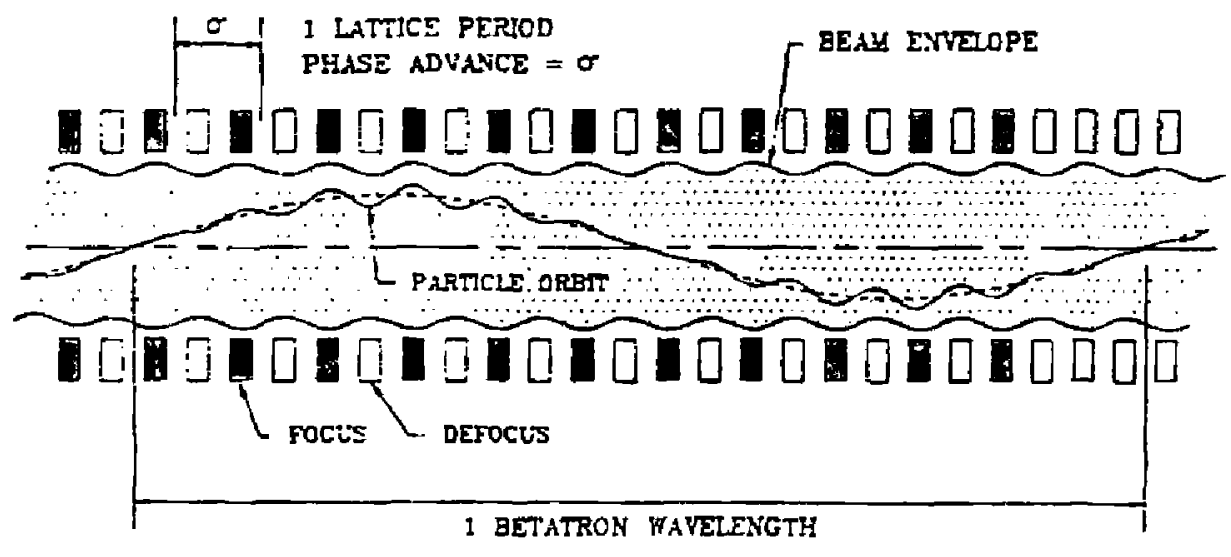

Fig - 1 


\section{Experimental limits on beam stability in terms of $\sigma$ and $\sigma_{0}$}

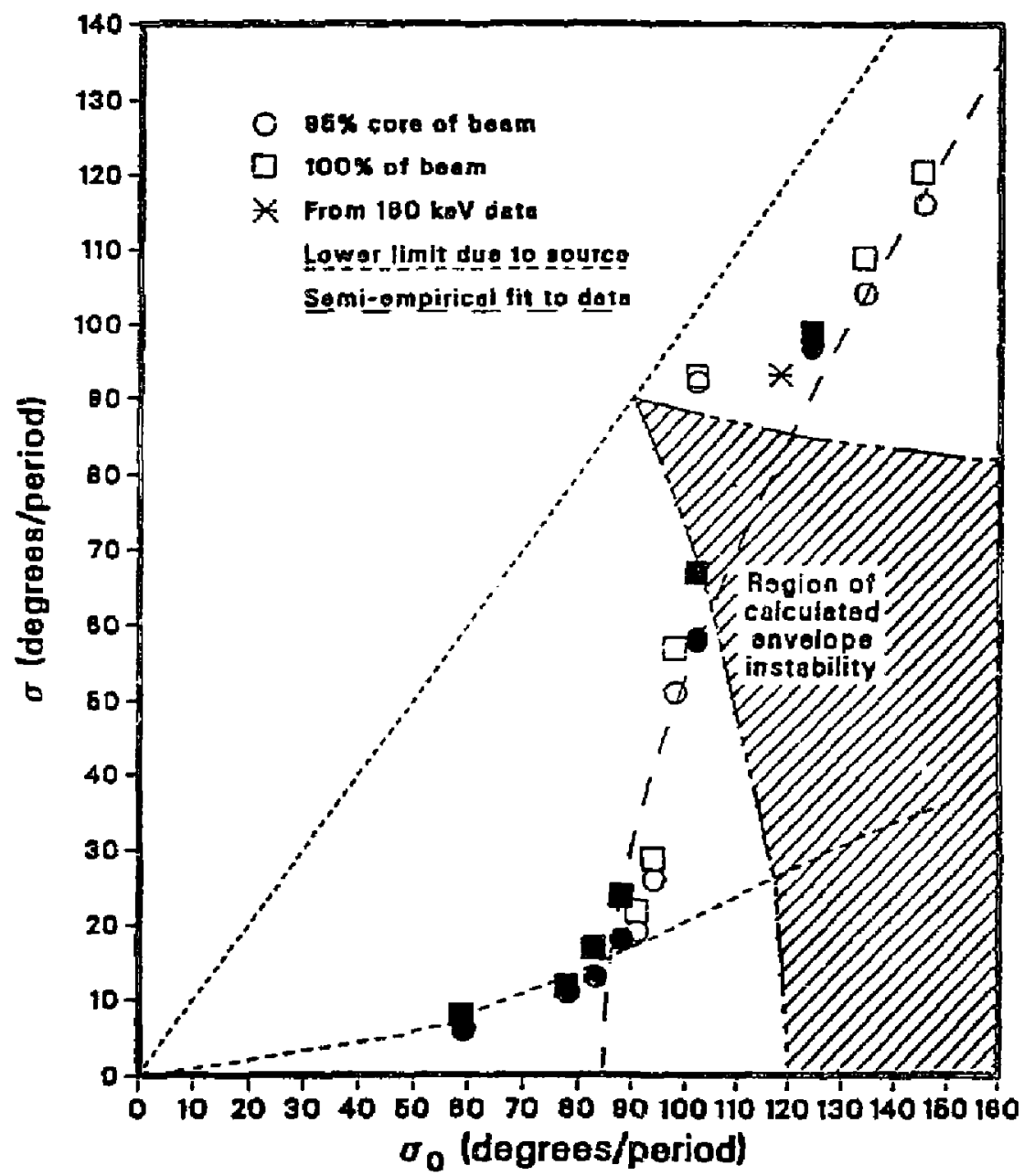

Fig. 2

XBL $865-1840$ 


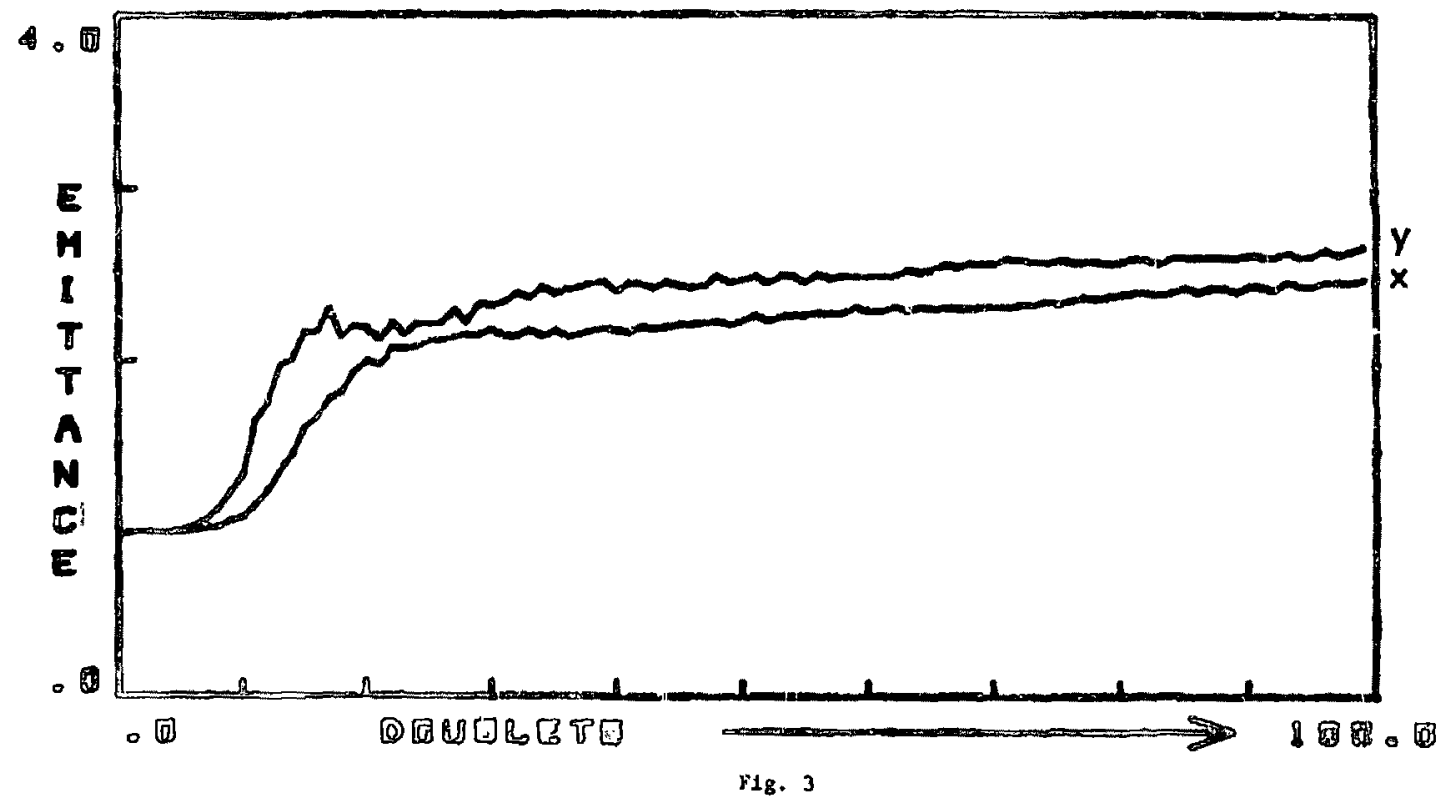



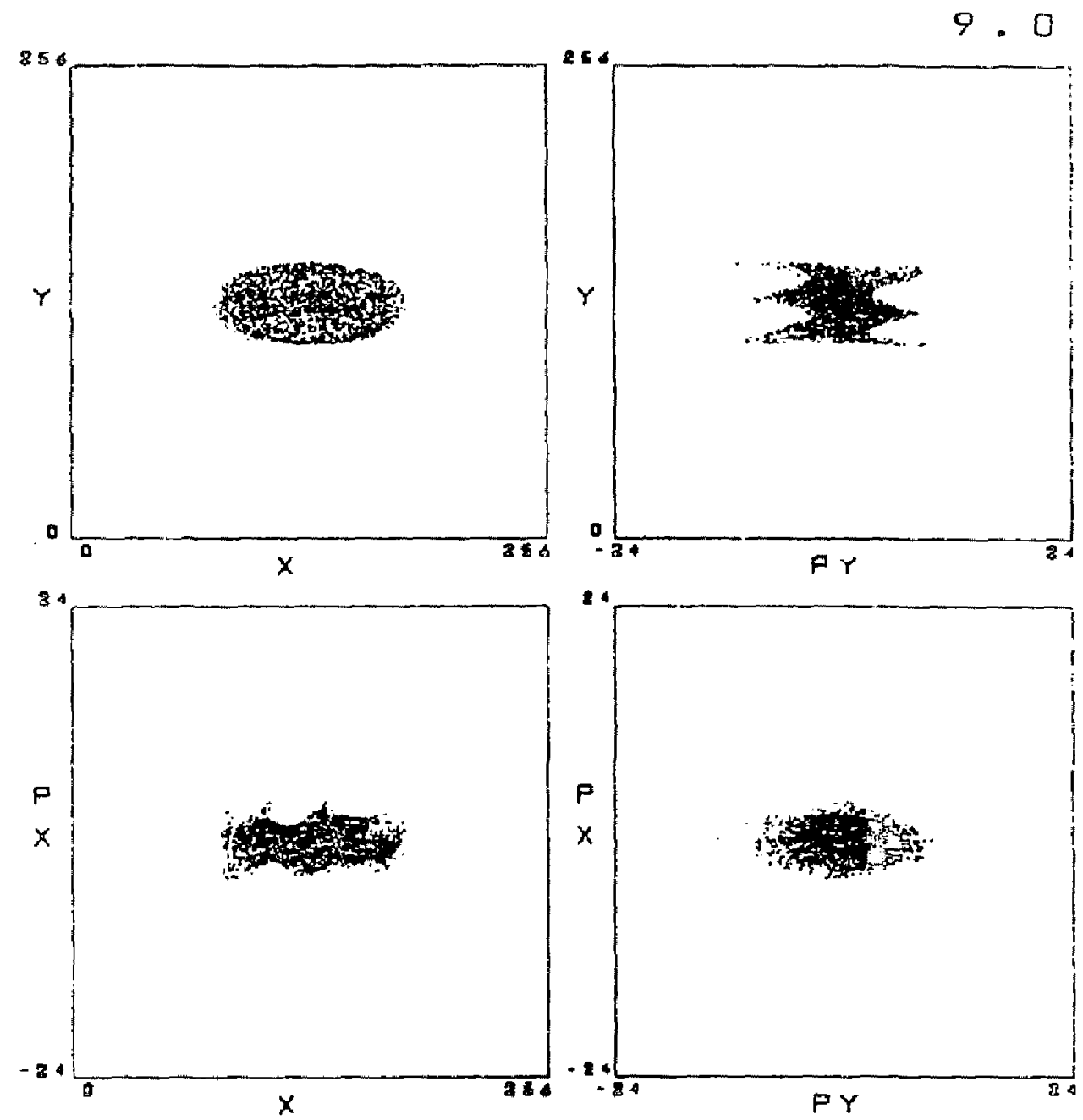

Fig. 4 


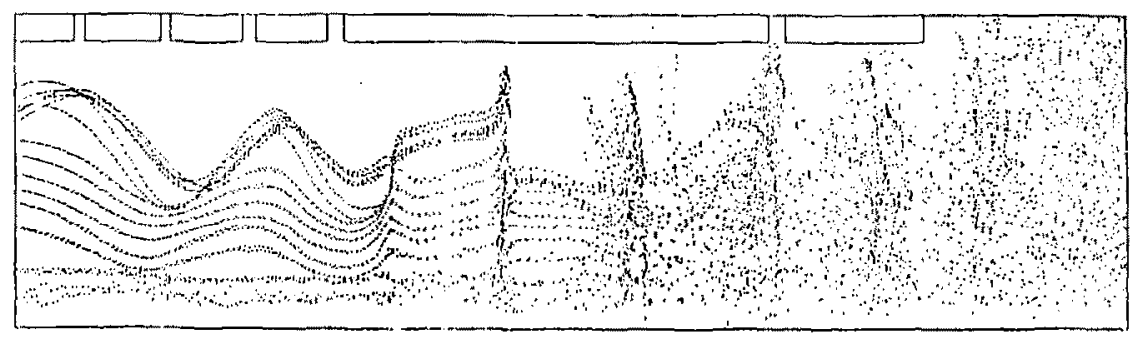

F1s. 5 


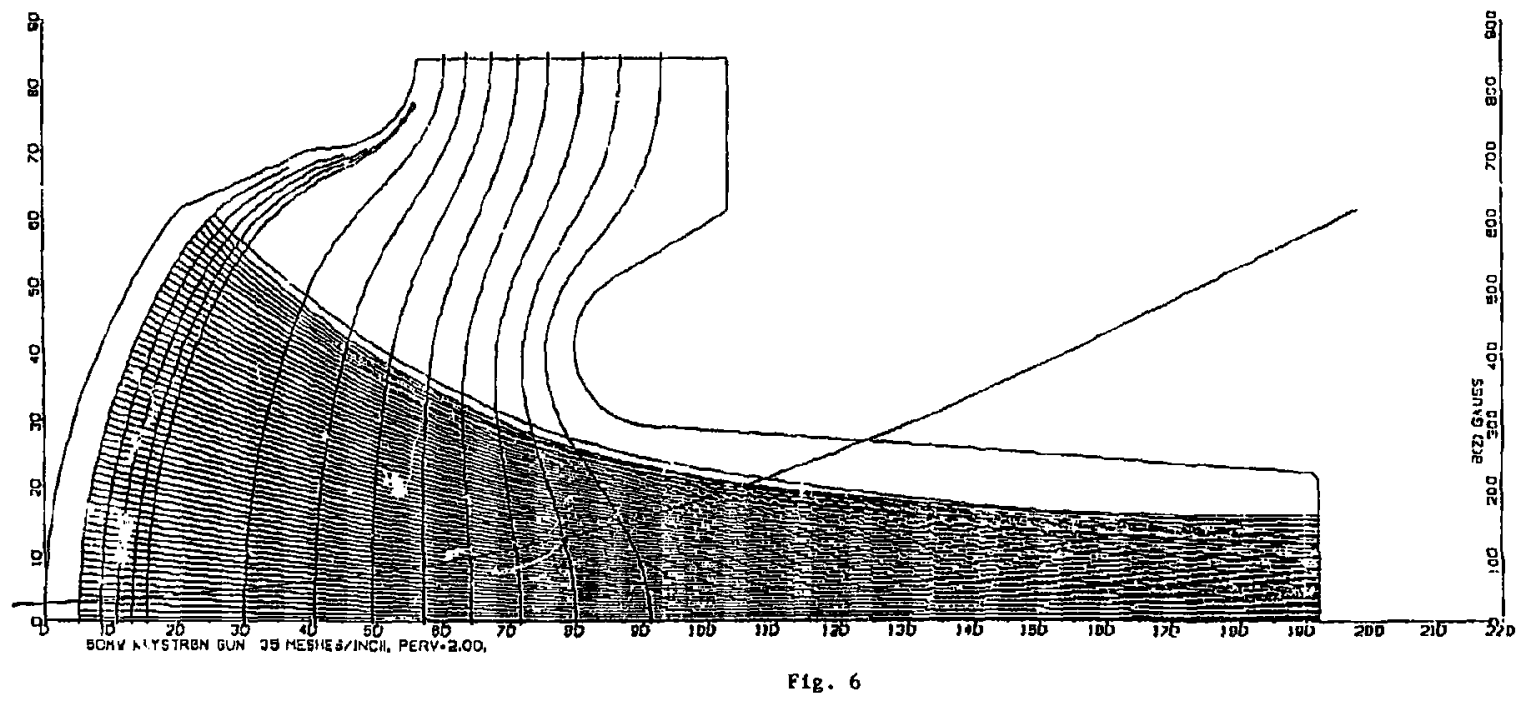




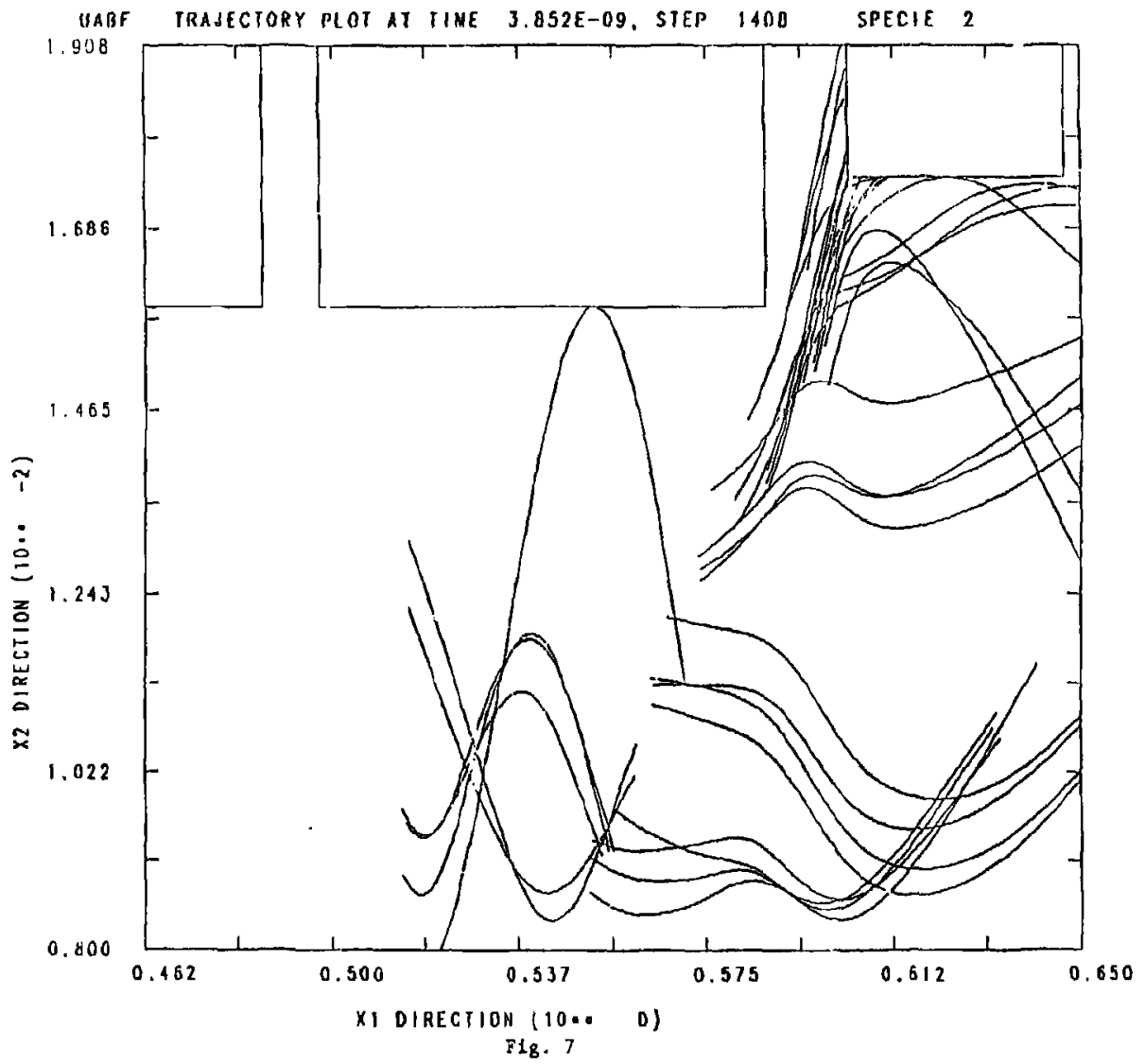




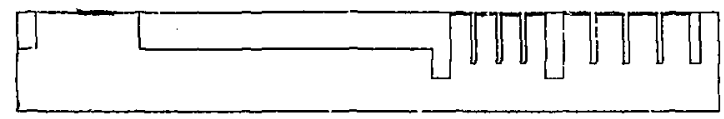

F1g. 8 

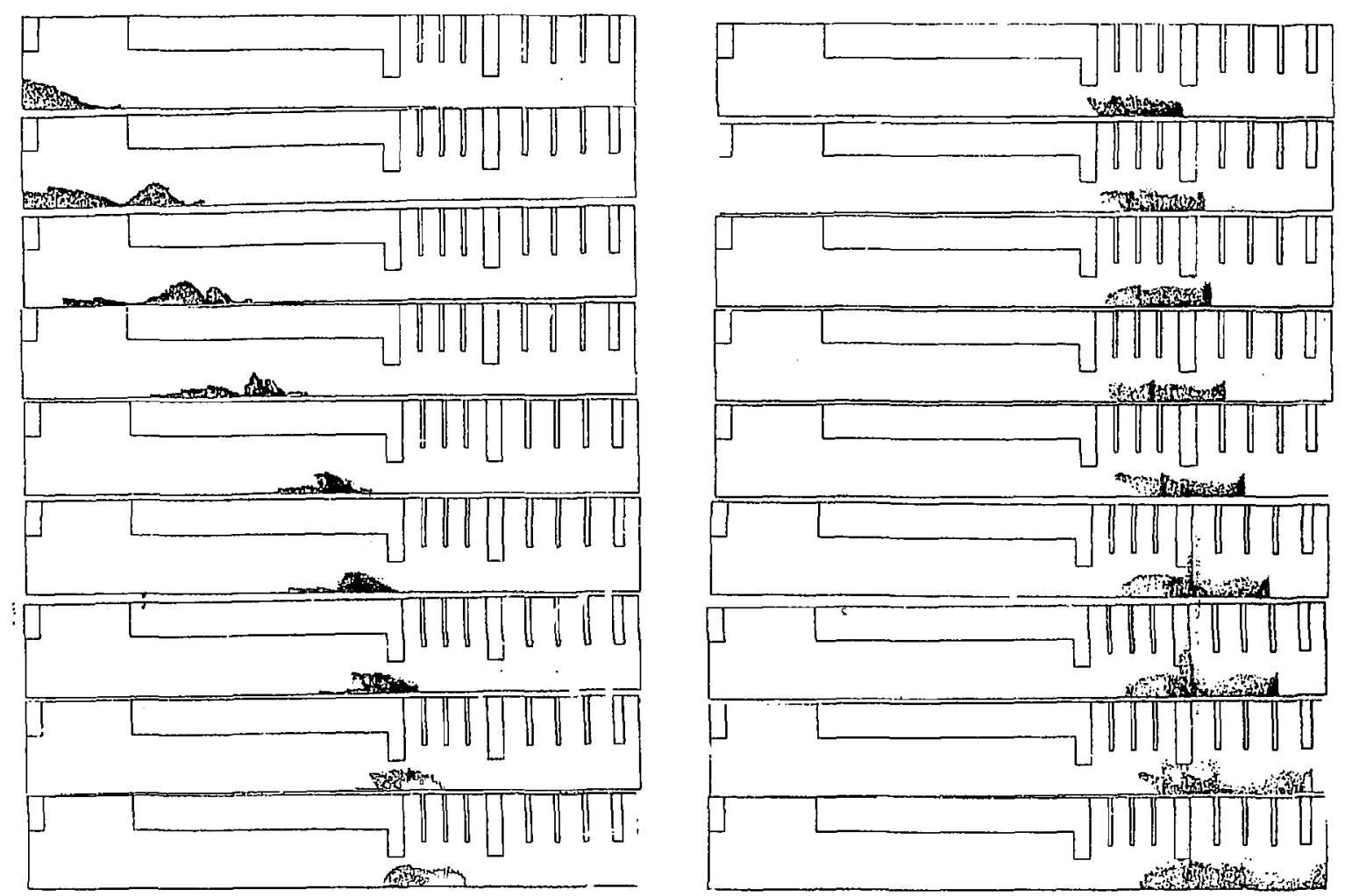

Fig. 9 


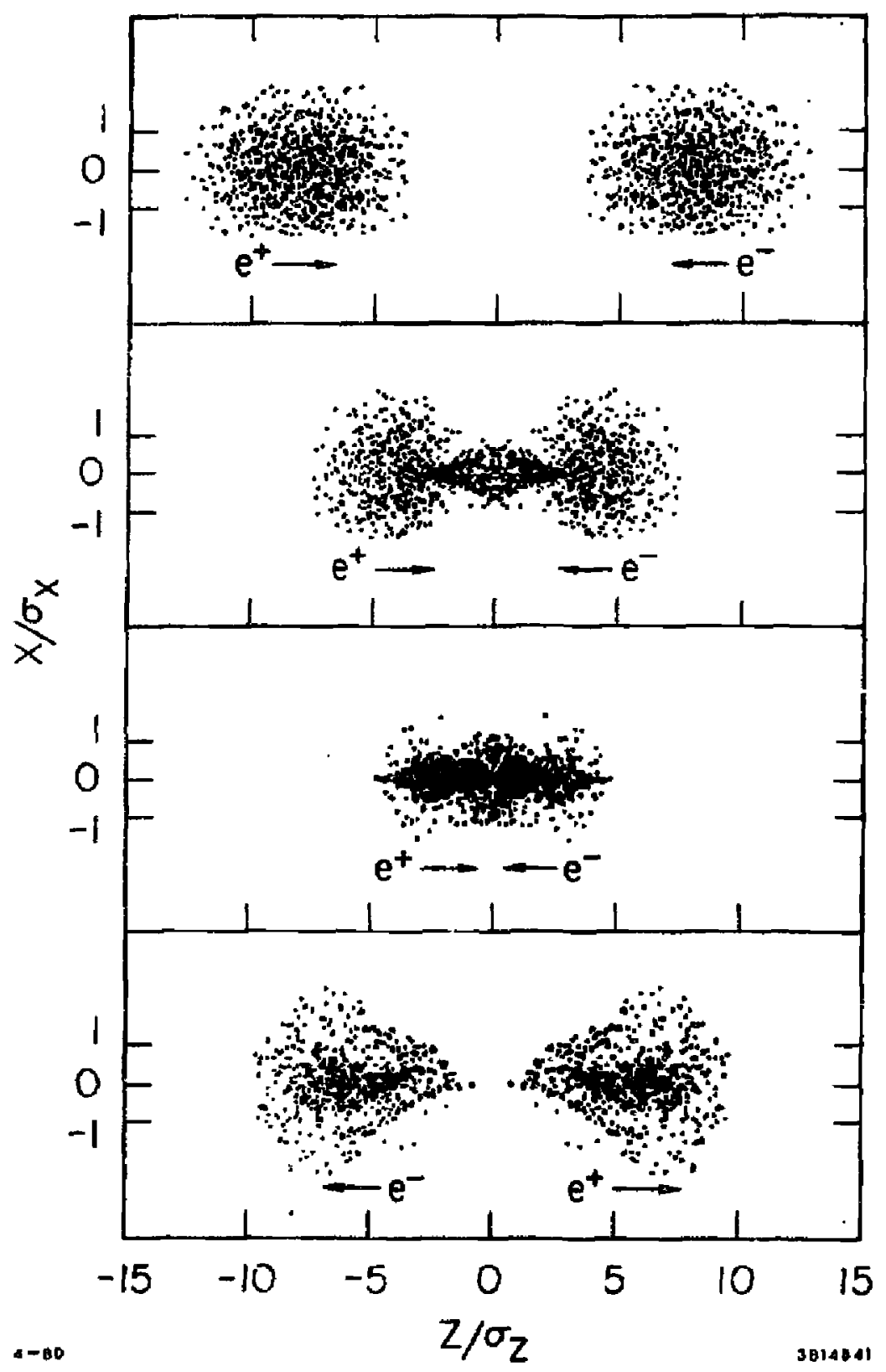

F18. 10 